\title{
DEVELOPMENT OF A PRESSURE MEASURING UNIT BASED ON A THERMAL CONDUCTIVITY GAUGE AND A LOW-COST EMBEDDED SOLUTION FOR MID-RANGE VACUUM APPLICATIONS
}

\author{
Albert-Zsombor FEKETE ${ }^{1}$, László JAKAB-FARKAS² \\ Sapientia Hungarian University of Transylvania, Faculty of Technical and Human Sciences, Târgu-Mureş, \\ Romania, \\ ${ }^{1}$ zsombor.fekete@tetronic.ro \\ ${ }^{2}$ jflaci@ms.sapientia.ro
}

\begin{abstract}
This study presents the development of a pressure measuring unit based on a Pirani gauge and a dedicated embedded system, incorporating a simple, low-cost practical solution for significantly reducing the various measurement altering factors, such as drifts, offsets and set point drifts. This is achieved by eliminating the conventional differential analogue signal processing stage and replacing it with a high resolution analog to digital converter. Therefore the goal was to minimize the number of the electronic components whose operation is influenced by variations in ambient temperature. The main topics discussed in the paper include the presentation of the measuring circuit 's configuration, the development of a low-cost embedded system, the calibration method and the solution implemented for eliminating the platforms limitation regarding the precision of the different mathematical calculations.
\end{abstract}

Keywords: vacuum pressure, thermal conductivity gauge, low-cost embedded solution, high resolution analog to digital converter, network connectivity.

\section{Introduction}

One of the most important elements in the construction of vacuum equipment is the evacuation system. Any minor malfunction or leak in the evacuation system could lead to an unwanted system shutdown and possibly to irreversible damage of the pumping units. In order to prevent this, one of the most important tasks in operating a high vacuum system [2] is the proper monitoring of the vacuum levels at different stages of the equipment.

\section{Thermal conductivity gauge}

In a mid-range vacuum line found, for example, between a turbo-molecular and a rotary pump the most common transducer type is the thermal conductivity or Pirani gauge. The sensor uses the dependence of thermal conductivity as a function of pressure [1]. The temperature of the sensor `s heated filament is an equilibrium temperature, which is set by the input and output heat balance. The thermal conductivity of the gas decreases with decreasing pressure, thus the thermal equilibrium temperature shifts causing changes in the resistance of the filament as well. This change of resistance can be attributed to the pressure change, and therefore is an indirect indication of its value [1, 2].

The gauge can be used in a relatively wide range of pressures (5-10-4 Torr). The relatively simple and robust construction of the gauge results in the need of little or no maintenance. It is advised to recalibrate the gauge yearly. 


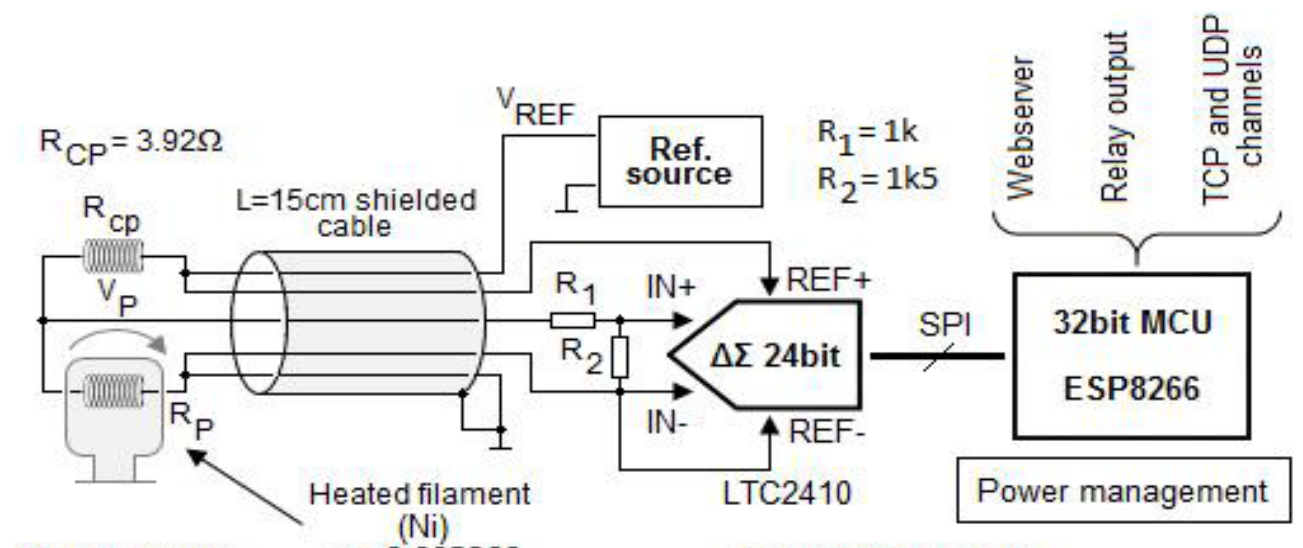

Pirani gauge $\quad \alpha=0.005866$

Embedded system

Figure 1. Simplified structure of the measuring circuit and the embedded system

\section{The embedded system}

The aim was to develop a low-cost embedded system (Figure 1.), which uses an LTC2410 delta-sigma type [3] analog to digital converter (ADC), connected directly to the terminals of the nickel Pirani filament $\left(\mathrm{R}_{\mathrm{p}}\right)$. This approach eliminates the conventional differential amplifier stage, thereby minimizing the number of active components that can introduce unwanted drifts and offsets. The full-scale error in this case is only $2.5 \mathrm{ppm}$ with a temperature dependent error drift of $10 \mathrm{nV} /{ }^{\circ} \mathrm{C}[3]$.

The analog to digital converter is connected to an 80 MIPS, 32 bit ESP8266 type microcontroller (MCU) [4] through a synchronous serial interface (SPI). The sampling time is set to 500ms and each conversion is triggered by the microcontroller.

The reference voltage for the ADC and the FAN type Pirani gauge is obtained with the help of an adjustable linear regulator.

The embedded system (Figure 2.) features wireless network connectivity, enabling multiple outputs in the form of TCP and UDP channels, and an embedded Web-server for remote access. Therefore the unit can act as an independent sensor connected to an Ethernet based process control network [5].

\section{Measurements and calibration}

The calibration process was effectuated with a full-range, PKR251 type pressure gauge from

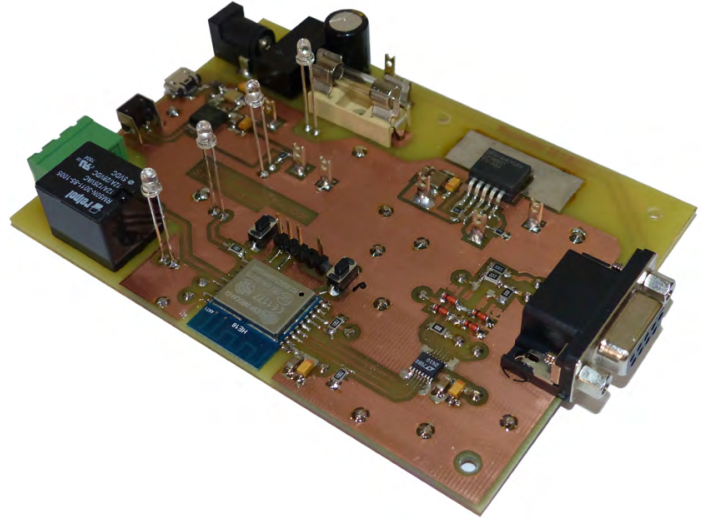

Figure 2. Prototype of the developed embedded system without enclosure

Pfeiffer Vacuum [6] with a global accuracy of $\pm 30 \%$.

Both gauges were connected to a vacuum chamber in which the dynamic pressure is controlled by the gas admission and evacuation. Measurements were performed for a gas mixture formed by $\mathrm{q}_{\mathrm{N} 2}=4 \mathrm{SCCM}$ and $\mathrm{q}_{\mathrm{Ar}}=6 \mathrm{SCCM}$ inlet mass flows, and with a 16bit ADC resolution.

The heat capacity and the thermal conductivity of the different gases varies [7], therefore the unit needs to be calibrated in the presence of the gases used in the actual application.

The temperature of the filament (1), as well as the temperature resolution (3) can be estimated by taking into consideration the dependence of the resistance on the temperature and the basic voltage equations of the presented electronic circuit. 


$$
T=\frac{\frac{V_{P} \cdot R_{C P}}{V_{R E F}-V_{P}}+R_{P}^{\prime}\left(\alpha \cdot T^{\prime}-1\right)}{\alpha \cdot R_{P}^{\prime}}
$$

where $R_{p},(3.72 \Omega)$ is the reference resistance of the Pirani filament at $T^{\prime}=30^{\circ} \mathrm{C}, \alpha$ is the temperature coefficient of resistance of the nickel, and $\mathrm{V}_{\mathrm{P}}$ is the voltage drop on the filament (2)

$$
V_{P}=\frac{A D C \cdot 0.5 \cdot V_{R E F} \cdot\left(R_{1}+R_{2}\right)}{R_{2} \cdot\left(2^{16}-1\right)}
$$

Based on equation (3), the resolution can be increased by raising the $\mathrm{V}_{\mathrm{REF}}$ reference voltage.

$$
\Delta T=\Delta V_{P} \cdot \frac{R_{C P}}{V_{R E F} \cdot \alpha} \cdot \frac{1}{\left(1-\frac{V_{P}}{V_{R E F}}\right)^{2}}
$$

The value of the $\mathrm{V}_{\text {REF }}$ was practically determined so that at the lower end of the pressure range the $A / D$ conversions quantum equals with a $\Delta \mathrm{p}=1.5 \cdot 10^{-5}$ Torr pressure change. Thus, with the lowest pressure of $4 \cdot 10^{-4}$ Torr and the resulting $194 \mathrm{mV}$ reference voltage, the filament `s temperature (1) is $95^{\circ} \mathrm{C}$. When used with oil sealed rotary pumps, it is advisable to operate the gauge at the lowest possible temperature, because oil vapours can condensate on the surface of the hot filament, changing its heat transfer parameters.

With given system parameters, in every of the 12 pressure ranges the result of the A/D conversion was approximated in function of the pressure with first or second order polynomial functions (Table 1.).

We determined the accuracy of the various approximation functions implemented in the embedded system, as well as the different resolutions available in the pressure ranges presented.

The mean error and the variance $\left(\sigma^{2}\right)$ describe the deviation between the calculated pressure and the pressure measured by the calibration gauge. The results are shown in Table 1. and Figure 3.

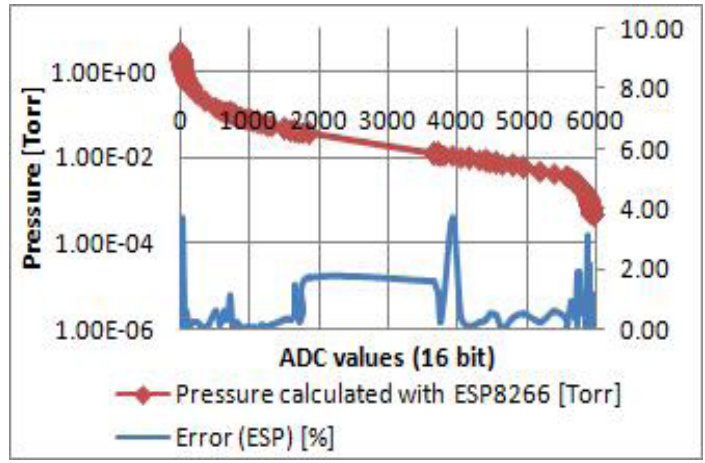

Figure 3. Pressure calculated with the ESP8266 embedded system and the obtained accuracy

\begin{tabular}{|c|c|c|c|c|c|c|}
\hline $\begin{array}{l}\text { Pressure range } \\
\text { [Torr] }\end{array}$ & \multicolumn{3}{|c|}{$\begin{array}{c}\text { Calibration functions: } p=f(x) \text { [Torr], where } \\
x=\text { ADC value }- \text { static offset }\end{array}$} & $\begin{array}{c}\text { AVG } \\
\text { Error } \\
\text { [\%] }\end{array}$ & $\begin{array}{c}\sigma^{2} \\
{[\%]}\end{array}$ & $\begin{array}{l}\text { Resolution } \\
\text { [Torr] }\end{array}$ \\
\hline $2 \div 1$ & $7.8046 \cdot 10^{-4} \mathrm{x}^{2}$ & $-6.5190 \cdot 10^{-2} \mathrm{x}$ & +2.5216 & 1.25 & 0.74 & $5.64 \cdot 10^{-2}$ \\
\hline $1 \div 8 \cdot 10^{-1}$ & & $-9.5460 \cdot 10^{-3} \mathrm{x}$ & +1.4252 & 0.25 & 0.01 & \multirow{4}{*}{$8.41 \cdot 10^{-3}$} \\
\hline $8 \cdot 10^{-1} \div 6 \cdot 10^{-1}$ & & $-5.1364 \cdot 10^{-3} \mathrm{x}$ & +1.1067 & 0.26 & 0.05 & \\
\hline $6 \cdot 10^{-1} \div 3 \cdot 10^{-1}$ & $1.0198 \cdot 10^{-5} \mathrm{x}^{2}$ & $-5.5330 \cdot 10^{-3} \mathrm{x}$ & +1.0323 & 0.18 & 0.01 & \\
\hline $3 \cdot 10^{-1} \div 1 \cdot 10^{-1}$ & $4.5231 \cdot 10^{-7} \mathrm{x}^{2}$ & $-7.4903 \cdot 10^{-4} \mathrm{x}$ & $+4.0840 \cdot 10^{-1}$ & 0.48 & 0.03 & \\
\hline $1 \cdot 10^{-1} \div 7 \cdot 10^{-2}$ & $1.0020 \cdot 10^{-7} \mathrm{x}^{2}$ & $-2.7747 \cdot 10^{-4} \mathrm{x}$ & $+2.4969 \cdot 10^{-1}$ & 0.08 & 0.01 & \multirow{3}{*}{$7.30 \cdot 10^{-5}$} \\
\hline $7 \cdot 10^{-2} \div 4 \cdot 10^{-2}$ & $3.6922 \cdot 10^{-8} \mathrm{x}^{2}$ & $-1.4581 \cdot 10^{-4} \mathrm{x}$ & $+1.8102 \cdot 10^{-1}$ & 0.24 & 0.02 & \\
\hline $4 \cdot 10^{-2} \div 1 \cdot 10^{-2}$ & $4.4449 \cdot 10^{-9} \mathrm{X}^{2}$ & $-3.7522 \cdot 10^{-5} \mathrm{x}$ & $+8.9687 \cdot 10^{-2}$ & 1.35 & 0.79 & \\
\hline $1 \cdot 10^{-2} \div 3 \cdot 10^{-3}$ & $6.8013 \cdot 10^{-10} \mathrm{x}^{2}$ & $-1.0591 \cdot 10^{-5} \mathrm{x}$ & $+4.1463 \cdot 10^{-2}$ & 0.37 & 0.05 & \multirow[t]{2}{*}{$5.03 \cdot 10^{-6}$} \\
\hline $3 \cdot 10^{-3} \div 1 \cdot 10^{-3}$ & $-1.247 \cdot 10^{-8} \mathrm{x}^{2}$ & $+1.3696 \cdot 10^{-4} \mathrm{x}$ & $-3.7305 \cdot 10^{-1}$ & 1.47 & 0.81 & \\
\hline $1 \cdot 10^{-3} \div 6 \cdot 10^{-4}$ & & $-8.9454 \cdot 10^{-6} \mathrm{x}$ & $+5.3825 \cdot 10^{-2}$ & 0.53 & 0.06 & \multirow{2}{*}{$1.51 \cdot 10^{-5}$} \\
\hline $6 \cdot 10^{-4} \div 4 \cdot 10^{-4}$ & & $-6.9886 \cdot 10^{-8} \mathrm{x}$ & $+5.3825 \cdot 10^{-2}$ & 0.51 & 0.01 & \\
\hline
\end{tabular}

Table 1. Pressure calibration functions for FAN Pirani sensor with a $194 \mathrm{mV}$ reference voltage, a 16bit resolution, and a gas mixture of $N_{2}$ and $\mathrm{Ar}$ 


\section{Software implementation}

Although the MCU features a 32bit platform, the development environment limits both double and float types to only 7 decimals. As a result, the accuracy of the various calibration functions decreases significantly.

The solution proposed for the presented problem assumes the use of a dedicated library [8], which extends the number of decimals to 16. On the downside the system requirements such as the RAM usage and the CPU processing time are increased. The maximum execution time is $4 \mathrm{~ms}$, which is only $0.8 \%$ of the sampling time.

\section{Conclusions}

The developed system offers a relatively simple and low-cost solution for measuring the pressure of a mid-range vacuum line. It can be used for a wide range of different Pirani gauges and the working parameters can be optimized according the function of the field of application. With the presented solution, the unit achieves an adequate resolution for a wide range of applications.

The embedded system serves as a vital safety element, being capable of sensing vacuum leakages and evacuation malfunctions.

\section{Acknowledgement}

The authors thank István Szöllösi and the members of the Thin Solid Films Research Group (TSFRG) from the Sapientia Hungarian University of Transylvania, for their contributions to the presented development work.

\section{References}

[1] P. K. Weng, J. S. Shie: Micro-Pirani vacuum gauge. Review of Scientific Instruments, 65/2. (1994) 492-499. https://doi.org/10.1063/1.1145163

[2] T. Brun, D. Mercier, A. Koumela, C. Marcoux, L. Duraffourg: Silicon nanowire based Pirani sensor for vacuum measurements. Applied Physics Letters, 101/18. (2012) 183506. https://doi.org/10.1063/1.4765665

[3] Linear Technology, 24-Bit No Latency $\Delta \Sigma^{\mathrm{TM}} A D C$ with Differential Input and Differential Reference. LTC2410 datasheet, 2000 (REV A).

[4] Espressif, ESP-WROOM-02. ESP8266 datasheet, 2017 (version 2.5).

https://www.espressif.com/en/products/hardware/esp-wroom-02/overview

[5] A. Zs. Fekete, A. Kelemen, L. Jakab-Farkas: Multilevel Distributed Embedded System for Control of the DC Magnetron Sputtering Process. Acta Universitatis Sapientiae Electrical and Mechanical Engineering, 9 (2017) 43-55. https://doi.org/10.1515/auseme-2017-0009

[6] Pfeiffer Vacuum, Compact Full Range ${ }^{\mathrm{TM}}$ Gauge, PKR251 datasheet, 2008-04.

[7] K. Toshio, H. Tadahiko, N. Yohta, T. Hiroyuki: Sensitivity Coefficients of Pirani Gauge for Various Atoms and Molecules. Japanese Journal of Applied Physics, 37/1. (1998) 369. https://doi.org/10.1143/JJAP.37.369

[8] N. Gammon, "BigNumber" library. Internet: https://github.com/nickgammon/BigNumber (accessed 14 February, 2018). 